\title{
Werner Kallmeyer
}

\section{Stadtsprache als ein Schwerpunkt soziolinguistischer Forschung in Europa}

\section{Das gegenwärtige Interesse der Soziolinguistik an der Stadt}

Die Stadt ist in den letzten Jahren verstärkt Gegenstand der Soziolinguistik in Europa geworden. So erscheint es zumindest aus deutscher Sicht, weil im deutschen Sprachraum einerseits aufgrund der Wissenschaftsentwicklung ein deutliches Defizit in der Behandlung städtischer Verhältnisse entstanden war (vgl. Radtke 1972; Dittmar/Schlieben-Lange 1982 b) und weil hier andererseits seit einigen Jahren ein markanter Wechsel eingetreten ist. Es gibt deutliche Phasenverschiebungen zwischen den einzelnen europäischen Ländern und Sonderentwicklungen, aber insgesamt verdichtet sich das Bild. Davon zeugen Tagungen und Kolloquien, Themenbände von Zeitschriften oder Reihen, ${ }^{1}$ Bestandsaufnahmen zur Stadtsprachenforschung wie die von Dittmar/Schlieben-Lange (1982b) und der als Ergänzung dazu von Brang vorgelegte Forschungsbericht zum slavischen Sprachraum sowie eine größere Zahl von Stadtforschungsprojekten aus der jüngsten Zeit (vgl. weiter unten).

In diesem Zusammenhang werden Forderungen nach einer weitergehenden Abstimmung und Verzahnung auf diesem Arbeitsgebiet laut. So weist z. B. Brang darauf hin, daß ,,eine gewisse Koordination im nationalen und übernationalen Rahmen dringlich zu wünschen (ist), nicht zuletzt im Hinblick auf die Vergleichbarkeit der Ergebnisse und im Interesse der Erarbeitung einer möglichst einheitlichen Terminologie“ (1986, 166). Und Dittmar/Schlobinski sehen den interurbanen Vergleich in Europa als .,ein dringendes soziolinguistisches Desiderat der nächsten zehn Jahre" an.

Für die allmähliche Verdichtung und ggf. Bündelung der Forschungsaktivitäten in der Stadtsprachenforschung gibt es grundsätzlich wohl zwei Klassen von Motiven, und zwar wissenschaftsinterne (a) und eher externe, gesellschaftspolitische (b):

(a) Es geht um die Fortsetzung, Wiederaufnahme oder auch Reorganisation eines langfristigen Forschungsprogramms der Sprachwissenschaft. Die Untersuchung der Sprachunterschiede innerhalb des Geltungsbereiches einer Standardsprache ist seit dem Beginn der Dialektologie Programm, und auch die Erforschung nicht nur der unter-

\footnotetext{
1 U.a. die Tagungen des Instituts für deutsche Sprache in Mannheim 1981 zum Thema „.Mehrsprachigkeit in der Stadtregion" (vgl. Bausch 1982) und zur Stadtsprachenforschung in Berlin 1984 (vgl. Dittmar/Schlobinski 1987), auch Kolloquien zur Ortssprachenforschung wie das in Bonn 1982 (vgl. Besch/Mattheier 1985) gehören zumindest ausschnittweise in diesen Kontext (vgl. weiter unten); siehe weiter das Themenheft „Sociolinguistics in France: Current research in urban settings“ des „International Journal of the Sociology of Language“ (vgl. Tabouret-Keller 1985) oder den Band zur „Sociolinguistica urbana in Italia e Europa occidentale“ (vgl. G. Klein 1987).
} 
schiedlichen Sprachen, sondern auch der Sprachunterschiede innerhalb einer umgrenzten Population wie einer Ortschaft oder Stadt ist schon früh formuliert und teilweise auch praktiziert worden. Die Wissenschaftsentwicklung ist allerdings in den einzelnen Ländern unterschiedlich verlaufen und ist $z$. T. auch mit Traditionsbrüchen verbunden. Die neueren Bestandsaufnahmen zur Entwicklung und Aufgabenstellung der Stadtsprachenforschung sowie zur Ortssprachenforschung zeichnen sowohl die Traditionsbildung als auch die Traditionsbrüche und Widersprüche der Entwicklung nach.

Dittmar/Schlieben-Lange (1982b) weisen besonders auf die Leistungen der Stadtsprachenforschung im romanischen Sprachraum hin, die sich kontinuierlich in bezug auf die Sprachatlanten, insbesondere für den italienischen Sprachraum, entwickelt habe. Im romanischen Sprachraum gibt es z. B. die frühen, dort auch traditionsbildenden Monographien von Silvioni über Mailand (1884), Rousselot über Cellefrouin in der Charente (1891) oder Gauchat über Charmey (1905) (vgl. dazu Dittmar/Schlieben-Lange 1982 b, 55f.). Die Arbeit von Gauchat widerlegte die erwartete sprachliche Einheit innerhalb eines dafür besonders prädestiniert erscheinenden Ortes völlig. Und es gibt die programmatischen Äußerungen z. B. von Jaberg zum Sprach- und Sachatlas für Italien und die Südschweiz 1933, der erläutert, daß der Atlas u. a. Informationen über die Beziehungen zwischen den ,,parlers directeurs" inklusive der Schriftsprache und der sozial untergeordneten Umgangssprachen liefern soll (vgl. insgesamt Dittmar/Schlieben-Lange 1982, 54ff.). Auch die derzeitigen Arbeiten am NADIR (Nuovo atlante dialettogico d'Italia per regioni) können hinsichtlich der Berücksichtigung der Stadt und des Stadt-UmlandVerhältnisses als besonders interessant angesehen werden.

Für die germanistische Tradition rekonstruiert z. B. Wiesinger (1985) die Widersprüche in den frühen dialektologischen Programmen von Wegener und Kaufmann zwischen der prinzipiellen Wahrnehmung der internen sprachlichen Variation und der durch die junggrammatische Theorie inspirierten Empfehlung für die Forschung, die Ortssprache als eine festgefügte Einheit zu beschreiben $(1985,29 \mathrm{ff}$.). In der Orts- wie in der Stadtsprachenforschung, die in den 20er Jahren verstärkt Interesse findet, gibt es immer wieder Arbeiten, die vorbildhaft sein könnten, aber nicht wirklich traditionsbildend geworden sind. So wie z.B. bei der Ortssprachenforschung die Arbeit von Enderlin (1910) über „Die Mundart von Kesswil in Oberthurgau“ nach dem Vorbild der Arbeit von Gauchat über Chamey (1905) sehr ,fortschrittlich“ war in der Annäherung an die komplexe sprachliche Wirklichkeit einer Ortsgemeinschaft, aber keine weitere Beachtung fand (vgl. Wiesinger 1985, 34f.), so gibt es Arbeiten wie die von Sexauer (1927), in der die Varietäten unterschiedlicher sozialer Gruppen von der alteingesessenen Landbevölkerung in den eingemeindeten Ortsteilen über die eingesessene und neu zugezogene Arbeiterschaft und die Gruppe der aufstrebenden Geschäftsleute bis hin zu den Zugewanderten berücksichtigt werden. Aber viele Stadtsprachenmonographien der 30er Jahre sind demgegenüber wiederum relativ ,konservativ“ (vgl. die Hinweise auf einige Arbeiten in Dittmar/Schlieben-Lange 1982b). Dasselbe gilt z. B. für die von Maas (1986, $37 \mathrm{ff}$.) aufgezeigten Entwicklungen ethnographischer Ansätze in der Dialektologie. Er hebt insbesondere die Arbeit von Mitzka über den Danziger Raum hervor (1928), in der zur Erklärung des Sprachausgleichs bereits eine Verbindung von sprachlichen und sozialstrukturellen Konzepten und Konzepten kultureller Praxis angelegt ist, wie sie in den 
letzten Jahren z.B. Gegenstand der Soziologie Bourdieus geworden ist. Mitzka erklärt den stattfindenden Sprachausgleich damit, daß ein einheitlicher sprachlicher Markt entsteht, auf dem die Beteiligten den Mehrwert oder Minderwert der Ausdrucksweisen in ihrer Praxis erfahren (Maas 1986, 40). Es scheint, daß es im deutschen Sprachraum eher Ansätze zu einer empirischen Tradition gibt, aber keine theoretische Formulierung eines Forschungsprogramms zur Erfassung der sozialen Differenzierung der Sprache wie etwa im romanischen Sprachraum.

In Rußland formuliert Larin 1926 ein Programm ,,zur linguistischen Charakterisierung der Stadt", in dem darauf hingewiesen wird, daß die Entwicklung der Literatursprache soziologisch gedeutet werden müsse und daß dies nicht möglich sei, .,solange ihr unmittelbares linguistisches Milieu nicht erforscht wird, d.h. die übrigen Typen der Schriftsprache und alle Varietäten der Umgangssprache des städtischen Kollektivs“ (vgl. Brang 1986, 142). Es scheint aber so, daß in der Weiterentwicklung und empirischen Umsetzung dieser Programme als ein wesentliches Hindernis die Orientierung hin auf die Norm der Literatursprache wirkt: Brang berichtet, da $\beta$ die einschlägigen Untersuchungen der 70er und beginnenden 80er Jahre die literarische Umgangssprache, d.h. die „ungezwungene Rede von Trägern der Literatursprache" erfaßten und nicht die alltägliche Sprache unterschiedlicher sozialer Gruppen in der Stadt (vgl. Brang 1986, 146).

Einen entscheidenden Entwicklungsschub bekam international die soziolinguistische Erforschung der Stadt in den 60er Jahren durch die Variationsanalyse von Labov (insbesondere die bahnbrechende Arbeit von 1966 über den Sprachgebrauch von New York City) und die sprachsoziologischen und -psychologischen Untersuchungen der Mehrsprachigkeit in New York durch Fishman. Als dritten wesentlichen Ansatz, der insbesondere durch die Arbeiten von Gumperz soziolinguistisch wirksam wurde, ist die Ethnographie des Sprechens zu nennen, die tendenziell für die Erforschung kleinerer Gemeinschaften von Bedeutung ist, aber eben auch von kleineren Gemeinschaften im städtischen Rahmen. Der Einfluß dieser Ansätze geht darauf zurück, daß hier mit expliziten soziologischen Konzepten (wie Schicht, Netzwerk, Domäne, Sozialökologie), mit elaborierten empirischen Verfahren (Stichproben, Tests, teilnehmender Beobachtung) und zentralen linguistischen Konzepten (z. B. Labovs Variablenregel, Gumperz Konzeption des Codeswitch) die sprachliche Variabilität in der Stadt angegangen wurden. Je nach vorhandenen „ortsansässigen“ Traditionen verlief die Rezeption unterschiedlich, insgesamt prägten diese Ansätze aber auch den Stil neuerer Arbeiten der Soziolinguistik der Stadt in Europa. Die Rückbesinnung auf vorhandene Traditionen in den Bestandsaufnahmen der letzten Jahre hat ihren tieferen Grund wohl darin, daß in der Soziolinguistik der Stadt (und des Ortes) erneut programmatisch wichtige Entwicklungsschritte vollzogen werden (die z.T. auch den Charakter von Korrekturen oder Verhinderungen von Rückschritten im sprachwissenschaftlichen Forschungsprogramm haben können). ${ }^{2}$

2 Ein in dieser Hinsicht „klassischer“ Fall ist die Arbeit von Gauchat über Charmey (1905), auf die sich schon Labov bezogen hat, deren Bedeutung aus der Sicht der heutigen Soziolinguistik und Ortssprachenforschung hervorgehoben wird: „I think Gauchat's study must now be seen as a superb piece of sociolinguistic research. In fact, it is probably the first demonstration of the importance of age-grading as a mechanism in the transmission of linguistic change" (Romaine 1982 a, 15; vgl. auch Wiesinger 1985). Hier geht es um die Auseinandersetzung mit den relativ 
(b) Soziolinguistik ist immer durch die Auseinandersetzung mit gesellschaftlichen Problemen mit bestimmt worden. So wie die nordamerikanische Soziolinguistik unter dem Eindruck der drängenden Probleme der Stadtentwicklung entstanden (und gefördert) worden ist, die mit der Einwanderung und der Ghettobildung in den amerikanischen Städten zusammenhängen, und so wie sich die Beschäftigung mit den GastarbeiterProblemen in den verschiedenen europäischen Ländern natürlich aus der Wahrnehmung der neuartigen Problemlage (im wesentlichen in den Städten) entwickelt hat, oder wie sich die Mehrsprachigkeitsforschung in der Schweiz oder Belgien aus den spezifischen Problemen in diesen Ländern mit allen sprachpolitischen Implikationen inspiriert hat, so scheint ein neuer Schub des Interesses der Sprachwissenschaft an der Stadt mit dem neuen Interesse an städtischen Lebensformen und einer Renaissance der Stadtkultur zusammenzuhängen.

Das neue bzw. veränderte Interesse an der Stadt ist kein exklusiv sprachwissenschaftliches. Vielmehr hat die Soziolinguistik hier Anteil an einer Interessenlage, die sich in der Soziologie in Projekten zur Wohnquartierforschung niedergeschlagen hat (vgl. z. B. Bodzenta/Speiser/Thum 1981) und in der Sozialpsychologie in Projekten zur Stadtwahrnehmung und Identifizierung mit der Stadt (vgl. Graumann/Schneider 1987). Nach der Subkulturforschung anhand städtischer Gruppen (zu der in England z. B. das Center for Contemporary Cultural Studies in den $70 \mathrm{er}$ Jahren wichtige Beiträge vorgelegt hat $)^{4}$ erscheint die Ausrichtung auf die Stadt u. a. auch in der deutschen Kulturwissenschaft (das Thema des Volkskundekongresses 1983 in Berlin lautete: „Großstadt. Aspekte empirischer Kulturforschung“; vgl. Kohlmann/Bausinger 1985). ${ }^{3}$

Gerade auch im Hinblick auf eine weitergehende Verdichtung und Verzahnung der soziolinguistischen Stadtforschung in Europa scheint es wichtig zu sein, die unterschiedlichen Perspektiven, unter denen die Stadt in der Linguistik und anderen Disziplinen betrachtet wird, und die am Gegenstand .Stadt" behandelten grundsätzlichen theoretischen und methodologischen Fragen zu verdeutlichen, um das Potential der soziolinguistischen Stadtforschung abschätzen zu können. Im folgenden werde ich mich deshalb primär an diesen beiden Punkten orientieren.

\section{Aspekte/Perspektiven soziolinguistischer Stadtforschung}

Eine wichtige Aufgabe der soziolinguistischen Stadtforschung ist es, einen integrativen theoretischen Rahmen zu finden, der es gestattet, die unterschiedlichen Perspektiven auf

starken Annahmen von Labov über die Sprachgemeinschaft und ihre Homogenität (so Romaine; wobei die Ausgangslage ist, daß die englische Stadtsprachenforschung sich ganz strikt an das Labovsche Untersuchungsschema und seine Implikationen gehalten hat).

$3 \mathrm{Vgl}$. Tabouret-Keller über die Untersuchungen von Rebaudière-Paty über das Lothringer Industriegebiet und von Chauvin über Fos-sur-mer (1985). Tabouret-Keller stützt sich bei ihrem Abgrenzungsversuch auf Wohnform (Mietshäuser) und Erwerbsweise (Industriebetrieb vs. Landwirtschaft). Man könnte auch an die verschiedenen nationalen Varianten von „Silicon Valley“ denken. Das industrialisierte Dorf ist u. a. eine geläufige Entwicklungsstufe im raschen Wachstum der europäischen Städte in der Industrialisierungsphase in der zweiten Hälfte des 19. Jahrhunderts und am Beginn des 20. Jahrhunderts. 
die Stadt zu vermitteln und aufeinander zu beziehen. Dabei ist zu bedenken, daß viele Untersuchungen zu Erscheinungen des städtischen Lebens nicht explizit auf die städtische Struktur bezogen sind, aber eigentlich nur so zu verstehen sind. Das gilt für viele Arbeiten zur Mehrsprachigkeit (vgl. dazu auch Tabouret-Keller 1985), aber auch für viele Untersuchungen zur Population der Arbeitswelt (wie bei Donath/Pape/Roloff/ Schönfeld 1981 oder Senft 1982). Und es ist die vielfältige Typologie städtischer Siedlungs- und Lebensformen zu bedenken. Neben dem häufig idealtypisch erscheinenden Fall der aus ihrem Umland klar hervortretenen historisch gewachsenen Stadt mit einer eigenen Stadtsprache sind u. U. gerade auch die Grenzphänomene des Städtischen theoretisch aufschlußreich. Zu diesen Grenzphänomenen gehören einerseits Siedlungsformen wie die Ansiedlung von Arbeitern und Angestellten großer Industriewerke im ländlichen Kontext, wobei die Bewohner nach der Erwerbsform und nach der Lebensweise Städter sind, aber wesentliche Merkmale der Stadt wie z. B. ein Zentrum fehlen. ${ }^{4}$ Dazu gehören andererseits auch Ballungsräume ohne klares Zentrum wie das Ruhrgebiet (vgl. u. a. Thies 1982; Mihm 1985a) oder auch die Region von Städten wie London oder Paris, mit einem starken Gravitationszentrum und einer großen Zahl von Städten und Dörfern im Umfeld, mit der Tendenz zusammenzuwachsen, z. T. auch mit der Tendenz, eine funktionierende Gemeindestruktur und damit auch eine gewisse Eigenständigkeit zu erhalten (in den letzten Jahren verstärkt).

$\mathrm{Zu}$ den forschungsgeschichtlich wichtigsten unterschiedlichen Perspektiven auf die sozialen und sprachlichen Konstellationen in der Stadt gehören die folgenden:

- die sprachgeographische Einordnung der Stadt und das Stadt-Umland-Verhältnis (2.1.)

- die Stadt als Varietätenraum (2.2.)

- die Mehrsprachigkeit in der Stadt (2.3.)

- die Binnenstrukturen der Stadt (2.4.)

- Gruppen, Milieus, Subkulturen (2.5.)

- die Stadt als Raum interkultureller Kommunikation (2.6.)

- Sprachgeschichte der Stadt (2.7.)

Diese Aspekte zusammen ergeben so etwas wie das Profil einer ..vollständigen“ Stadtuntersuchung, das aber wohl in keinem konkreten Projekt umfassend und gleichgewichtig verwirklicht werden kann.

2.1. Die sprachgeographische Einordnung der Stadt und das Stadt-Umland-Verhältnis

Die dialektologischen Sprachatlanten liefern der Stadtsprachenforschung mit der sprachgeographischen Einordnung wichtige Voraussetzungen. Die Einordnung der Stadt in die

4 Neben Klassikern wie Blom/Gumperz 1972 zählen hierzu die schon in Dittmar/Schlieben-Lange 1982 b referierten englischen soziolinguistischen Arbeiten unter dem Einfluß des Labovschen Ansatzes wie Trudgill 1974 über Norwich (zur Literatur vgl. im einzelnen Dittmar/SchliebenLange). Für den deutschsprachigen Raum vgl. u. a. Dittmar/Schlobinski 1987 über Berlin. Denison et al. 1977 über Graz, Dressler/Wodak 1982 über Wien, Dyhr/Zint 1985 zu Flensburg. Brinkmann to Broxten 1986 zu Frankfurt, Auer/Mössle 1987 zu Konstanz, Kallmeyer i. V. zu Mannheim. 
Sprachregion wird im Prinzip in allen sozialdialektologischen Stadtsprachenuntersuchungen vorgenommen und für die Bestimmung der sprachlichen Rolle der Stadt benutzt (so z. B. von Auer/Mössle 1987 bei der Untersuchung zur Konstanzer Stadtsprache unter Bezug auf den Südwestdeutschen Sprachatlas). Die angemessene Berücksichtigung der Stadt stellt bekanntermaßen ein besonderes Problem für die Sprachatlanten dar (vgl. u. a. Radtke 1972 sowie Dittmar/Schlieben-Lange 1982 b). Die Schwierigkeit besteht darin, die sozialen Faktoren der Variation im Rahmen der sprachgeographischen Grundanlage zu berücksichtigen und die komplexen sprachlichen Verhältnisse in der Stadt, für die ja der regionale Bezug eine Einflußquelle neben anderen (wie Standardsprache bzw. überregionale Sprache, Migrantensprachen, überregionale Gruppenkulturen) ist, auf wesentliche, sprachgeographisch darstellbare Merkmale zu reduzieren. Das Auffinden von solchen Merkmalen, die auch die sprachlichen Verhältnisse der Stadt angemessen wiedergeben, ist eine Aufgabe gerade der Soziolinguistik der Stadt.

Für die Soziolinguistik der Stadt ist unter sprach- und sozialgeographischer Perspektive das Stadt-Umland-Verhältnis von besonderem Interesse. Verdichtete Beobachtungen im Umfeld der Stadt können genauere Aufschlüsse über die Ausdehnung der Stadt durch Sprach- und Kulturformen geben. Debus (1962) z. B. hatte bei der Betrachtung der Ausstrahlung von Städten wie Köln, Düsseldorf, Kassel oder Marburg darauf hingewiesen, daß auf der Grundlage der Daten der Wenker-Untersuchung um 1880 feststellbar ist, in welchem Umfang der Proze $\beta$ der Verstädterung vor der politischen Eingemeindung erkennbar ist (vgl. dazu auch Mattheier 1982). Verdichtete Untersuchungen des städtischen Umfeldes gehören im Prinzip allgemein zum Programm der Erhebungen zu den Sprachatlanten (vgl. z. B. die engmaschige Erhebung in der Straßburger Region im Rahmen des Elsässischen Sprachatlasses). Besonders interessant erscheinen in dieser Hinsicht zur Zeit die Arbeiten am NADIR (vgl. Sobrero 1985), weil hier Wanderungsbewegungen und Aktionsräume, Netzwerke und soziale Kategorisierung exemplarisch behandelt werden. Für die Behandlung des Stadt-Umland-Verhältnisses ergibt sich damit eine Verbindung zur Untersuchung von Urbanisierungseinflüssen in der Ortssprachenforschung (vgl. u.a. Gal 1979; Besch et al. 1981). Die sprachliche Auswirkung von Urbanisierungsprozessen in ländlichen Regionen sind auch Gegenstand der in Pedersen 1985 dargestellten skandinavischen Projekte. Hinsichtlich der Frage, wie weit die Stadt eigentlich reicht und wie die Prozesse der Stadtbildung verlaufen, zeigen die vorliegenden Untersuchungen unterschiedliche Konstellationen, u. a. in Abhängigkeit davon, ob sich eine ländliche Gemeinschaft durch Arbeits- und Einkaufspendeln allmählich für städtische Einflüsse öffnet (wobei sich der Wandel der Erwerbsform von der Landwirtschaft zur Industriearbeit als ein entscheidender Faktor für den Sprachwandel erweist, wie z. B. Gal 1979 nachweist), oder ob eine bereits urbane Population zuwandert und auf diese Weise eine Koexistenz von verschiedenen Kulturen im Rahmen von kleineren Ortschaften entsteht (mit unterschiedlichen Konsequenzen für die Definition der beteiligten Sprachen: Chauvin 1985 z. B. beschreibt für die Industrieansiedlung Fos-sur-mer mit einem dominanten Anteil von gehobenen, aufstiegs- und normorientierten Angestellten die Transformation der regionalen Sprache in einen Soziolekt; für das Umfeld deutscher Städte ist dagegen vielfach eine positive Zuwendung von zugewanderten Angehörigen der white-collar-Gesellschaft zum Lokalen zu beobachten). 
Dem Ausufern der Stadt durch die Urbanisierungsprozesse in ihrem Einzugsbereich steht das Fortbestehen des Dorfes und landschaftlicher Orientierungen in der Stadt gegenüber. Eine Komponente der Sprachgeographie innerhalb der Stadt sind die Spuren der Beharrung, vor allem in den städtischen Randbezirken mit eingemeindeten selbständigen Ortschaften (so in vielen dialektologischen Stadtsprachenuntersuchungen; für England vgl. Trudgill 1974), unter bestimmten Umständen existieren solche Sprachunterschiede aus vor-städtischer Zeit aber auch in weiten Teilen der Stadt (so weist Mihm für Duisburg weit zurückreichende Einflüsse aus der Geschichte der von der Stadt erfaßten alten Ortschaften nach; vgl. Mihm 1985b).

\subsection{Die Stadt als Varietätenraum}

Eine Kernaufgabe der Stadtsprachenforschung ist die Beschreibung der Sprachenkonstellation in der Stadt. Programmatische Äußerungen weisen darauf hin, daß Stadtsprache als ein Varietätenraum zu begreifen ist, der aus allen miteinander in Verbindung stehenden Sprachen und Sprachvarietäten gebildet wird (vgl. z. B. Hoffmann/Mattheier 1985, 1837). Im Rahmen einer solchen umfassenden Konzeption ist eine Teilaufgabe die Beschreibung der Stadtsprache als einer spezifischen Varietät bzw. als städtische Umgangssprache im Rahmen der sprachlichen Schichtung einer Stadt zwischen Dialekt und Standardsprache bzw. lokaler/regionaler und überregionaler Sprache. In mehr oder minder großem Umfang ist eine solche sozialdialektologische Beschreibung der städtischen Sprache Bestandteil der meisten Stadtforschungsprojekte.

Ein wesentliches Problem ist immer noch die Identifizierung und Abgrenzung von Varietäten städtischer Sprache. In der Diskussion um die „städtische Umgangssprache“ als eine durch Ausgleichsphänomene geprägte Sprachschicht zwischen Mundart und Hochsprache ist die Abgrenzung der Schichten immer ein Problem geblieben, und diese Schwierigkeiten haben vielfach zur Aufgabe des Schichtungskonzeptes zugunsten der von Labov vertretenen Kontinuumsvorstellung geführt. In der Diskussion um die konkurrierenden Modellvorstellungen des sprachlichen Kontinuums bzw. verschiedener Varietäten (Existenzformen) scheint es zur Zeit zwei gegenläufige Tendenzen zu geben. Auf der einen Seite ist offenbar die Variation zwischen Standardsprache und Dialekt besonders gut zu beschreiben auf der Grundlage des Kontinuumsmodells. Auf der anderen Seite ist es aber wohl so, daß zumindest subjektiv, bei der Selbst- und Fremdeinschätzung, das Kontinuum in „eigene“ und „fremde“ Sprache gegliedert wird bzw. in eine Zone der „normalen“, unmarkierten Sprache und Zonen der auffälligen, markierten Sprache (nach oben zum Standard und nach unten zum Dialekt; vgl. Rosenberg 1986). Die Gliederung des Kontinuums geschieht zweifellos soziozentrisch, und eine wichtige Aufgabe der Beschreibung ist sicher die Bestimmung der unterschiedlichen sozialen Perspektiven in Abhängigkeit von aktiven und passiven Sprachkenntnissen, Innen- und Außenorientierungen in bezug auf lokale Gemeinschaften usw. (vgl. Kallmeyer i.V.).

Eine an sich naheliegende Hypothese ist, daß die Verhältnisse je nach historischen Voraussetzungen und Stadtformen unterschiedlich liegen. Dressler/Wodak (1982) z. B. gehen zur angemessenen Berücksichtigung der Wiener Verhältnisse von einem Zweisprachenmodell mit österreichischem Standard-Deutsch und Wiener Mundart aus. Sie 
betrachten die sprachliche Situation in Wien als ein Beispiel für spezifisch europäische Verhältnisse im Unterschied zu den Verhältnissen in amerikanischen Städten, die durch hohe Mobilität der Bevölkerung geprägt sind und eher eine Kontinuumsvorstellung angemessen erscheinen lassen $(1982,366)$. Aber es bleibt natürlich die Frage, wie sich soziozentrische Sprachwahrnehmung und faktisches Variationsverhalten zueinander verhalten. Hier sind u. U. komplexere Verhältnisse möglich etwa derart, daß unterschiedliche Kategorien von Sprechern unterschiedliche Modelle als Bestandteil ihres sprachlichen Verhaltensstils praktizieren, u. U. sogar sich funktionsabhängig mal wie ein .,Kontinuums-Sprecher" und mal wie ein „Existenzform-Sprecher“ verhalten. ${ }^{5}$

Eine weitere zentrale Aufgabe ist die Beschreibung von sprachlichen Konvergenzund Divergenzvorgängen und deren Erklärung. Ein allgemeines Merkmal der komplexen städtischen Verhältnisse ist offensichtlich, daß sich unterschiedliche Prozesse der Durchsetzung von dominanten Normen bzw. der Anpassung an diese, der Beharrung und auch der produktiven Differenzierung überkreuzen. Die sprachlichen Unterschiede werden nicht geringer, sondern sie verlagern sich. Eine solche Verlagerung stellt z. B. die Tendenz dar, daß im Kontakt von Dialekt und Standardsprache insbesondere die Phonologie langfristig konvergiert, während sich in der Lexik große Unterschiede erhalten bzw. sich in Prozessen der sozialen Differenzierung neu entwickeln. Ein allgemeiner Befund ist auch, daß sich bei aufwärts mobilen Bevölkerungsschichten, die der negativen Bewertung ihrer Herkunftssprache entrinnen wollen, besondere Normenorientiertheit und zugleich Merkmale der Unsicherheit wie Hyperkorrekturen oder starke Schwankungen zwischen kontrolliertem und unkontrolliertem Sprechen zeigen. Ein Beispiel für dieses letzte Phänomen liefert die Untersuchung von Dressler/Wodak (1982) über das Verhalten von Angeklagten und Zeugen unterschiedlicher sozialer Herkunft in der Gerichtssituation.

Im Vordergrund stehen und entsprechend gut analysiert sind in den Stadtsprachenuntersuchungen zweifellos Phonetik/Phonologie, auch noch Morphologie/Syntax, weniger Lexik und ggf. Semantik und sehr wenig Pragmatik (vgl. dazu Dittmar/Schlieben-Lange 1982 b). Zur Zeit ist es noch so, daß Semantik und Pragmatik eher in den Ethno-Wissenschaften behandelt werden als in der Soziolinguistik. Diese praktiziert eigentlich einen relativ engen Begriff von Sprache bzw. von den sozial bedeutsamen Eigenschaften von Sprache. In den Ethnowissenschaften werden Kategoriensysteme und Handlungssysteme, Kommunikationsroutinen, Handlungs- und Textmuster (wie Spiele, Formen des Rechtsprechens, Zaubersprüche usw.) seit langem als Gegenstand angesehen. Es gibt gute Gründe dafür, diese Gegenstände auch in die soziolinguistische Stadtforschung zu integrieren. Ein wichtiger Gesichtspunkt in diesem Zusammenhang ist, daß sprachliche Eigenschaften von Sprechern immer als Teil eines in sich mehr oder weniger konsistenten sprachlichen Verhaltens auftreten, das insgesamt z.B. soziale Zugehörigkeit und bestimmte soziale Eigenschaften signalisiert (zur Bedeutsamkeit pragmatischer Normen als einem Gesichtspunkt dieser Verhaltensweisen vgl. auch weiter unten). Die Verbindung von Sprache und Sprechweise gehört ja auch zur landläufigen Vorstellung von

5 Einige Befunde im Mannheimer Projekt deuten darauf hin, daß es sowohl derartige Unterschiede des Verhaltensstils in einer Population als auch der Verhaltensweise einzelner Sprecher gibt. 
Stadtkultur: zum Berlinischen gehört die „Berliner Schnauze“, zum Mannheimerischen gehört die „Mannemer Gosch“ usw.

\subsection{Mehrsprachigkeit}

Neben der Variation auf der Achse zwischen Standard und Dialekt ist ein weiterer prägender Aspekt der städtischen Sprachenkonstellation die Mehrsprachigkeit unterschiedlicher Form und Herkunft. Es gibt einmal den Fall der Kopräsenz von mehreren Sprachen in Staaten mit mehreren staatlich anerkannten Sprachen, die auch regional verteilt sind, wobei die langfristig mehrsprachigen Städte an den innernationalen Sprachgrenzen liegen, und die Kopräsenz der Sprachen u. a. gestützt wird durch den Bezug auf das jeweilige .,Hinterland“, d. h. die entsprechende Sprachregion. Das ist z. B. der Fall in einer Reihe von Schweizer Städten oder in Belgien, insbesondere Brüssel. ${ }^{6}$

Ein anderer Fall ist die Überlagerung von regionalen Minderheitensprachen durch die dominante Staatssprache (vgl. z. B. Ladin 1982 und Gardener-Chloros 1985 zu Straßburg sowie Allardt et al. 1979 zur schwedischen Minderheit in Helsinki). In diesen Fällen spitzt sich die konfliktäre Sprachenkonstellation in den Städten im Zweifelsfall besonders zu wegen der Präsenz der dominanten Sprache in den politischen, administrativen und wirtschaftlichen Zentren.

Und schließlich gibt es die Mehrsprachigkeit aufgrund von überregionaler und internationaler Migration. Überregionale Migration ist z. B. ein relevanter Faktor in den norditalienischen Städten mit der Zuwanderung aus Süditalien (vgl. Dittmar/SchliebenLange 1982a), oder in Barcelona mit der Zuwanderung aus Andalusien. Bei den internationalen Wanderungsbewegungen ist die Arbeitsmigration der entscheidende Faktor, gefolgt von der Migration politischer Asylanten. Insbesondere die Gastarbeiter sind Gegenstand der Soziolinguistik geworden, wobei die Gastarbeiterstudien in Europa z.T. als Äquivalent zu den amerikanischen Einwandereruntersuchungen zu verstehen sind. Der Bezugsrahmen ist bei diesen Untersuchungen meistens nicht die Stadt, sondern eine Bevölkerungsgruppe mit spezifischen sprachlichen Defiziten, ihren Spracherwerbsprozessen, ihrer sozialen Benachteiligung. Aber als Folge von Segregationsprozessen finden diese Wanderungsbewegungen auch einen sozialgeographischen Niederschlag in der Stadt bis hin zur Ghettobildung. (Vgl. Berlin-Kreuzberg, arabische Stadtviertel oder Chinatown in Paris, Ausländerviertel in Südlondon usw.)

Insgesamt gilt für die verschiedenen Arten der städtischen Mehrsprachigkeit, daß die Stadt aufgrund ihrer Zentrumsfunktion sowie der innerstädtischen Verdichtungs- und Segregationsprozesse als Brennpunkt für soziale, sprachliche und politische Konstellationen fungiert, die in ihrer Existenz nicht an die Stadt gebunden sind. Die Ausprägung dieser Konstellationen sind dann aber wieder typisch städtisch.

Die Arbeiten zur stabilen Mehrsprachigkeit im staatlichen Rahmen knüpfen in der Regel an die Konzeption von Fishman zum Verhältnis von Diglossie und Mehrsprachig-

6 Vgl. u.a. die Arbeiten zu Brüssel von Nelde 1978, Baetens Beardsmore 1983 und Deprez/ Persoons 1983 sowie genereller Mattheier 1983, Kolde 1981 zu Biel und Fribourg, Lüdi et al. 1987 zu Neuchâtel. 
keit und zur Domänenverteilung von Sprachen an und versuchen, diese Vorstellung fortzuentwickeln. Eine Entwicklungsrichtung zielt darauf, die Komplexität der Verhältnisse zu rekonstruieren, die durch die Beteiligung von mehr als zwei Sprachen mit unterschiedlichen Hierarchisierungsachsen zwischen .,hoher" und .,niederer" Sprache entstehen. Ein Beispiel dafür ist Beatens Beardsmore (1983); hier wird von Analysen zur Sprachenlage in Brüssel berichtet, die sich dadurch auszeichnen, daß für Flamen und Wallonen jeweils eine dreistufige sprachliche Unterteilung gilt: für die Flamen gibt es die regionalen Dialekte, den ,zuidnederlands standaard“ und das „,algemeen Nederlands“; für die Wallonen gilt entsprechend die Abstufung von den regionalen Dialekten, dem regionalen Französisch und dem übernationalen Standardfranzösisch. In Abhängigkeit von der Herkunft, dem Alter und der Orientierung auf das flämische Hinterland oder die dominant französische Hauptstadt gibt es für verschiedene Bevölkerungsgruppen ganz unterschiedliche Verteilungen von Varietäten der beiden beteiligten Sprachen auf einer triglossischen Abstufung (supreme, hoch und niedrig). Dabei zeigt sich, daß unter dem Einfluß der neuen Höchstsprache ,.,algemeen Nederlands“ für die jüngere flämische Bevölkerung das Französische als L-Sprache hinter das regionale oder lokale Niederländisch rutscht. Die triglossische Struktur ist wahrscheinlich als eine Übergangsphase zu einer späteren stabilen Diglossie Niederländisch - Französisch für die flämischen Gruppen zu interpretieren. Solche Untersuchungen zeigen zugleich die Dynamik der Verhältnisse auf, die mit dem Konfliktcharakter der gesellschaftlich-sprachlichen Konstellation zusammenhängt und für welche die ursprüngliche Konzeption Fishmans zu starr ist. Generell scheint hier ein Ansatzpunkt für eine Diskussion um Konzepte wie ,Diglossie', ,Sprachkonflikt”, ,Dominanz' usw. gegeben (vgl. u.a. Dittmar/Schlieben-Lange 1982b zur katalanischen Soziolinguistik).

Bei der Untersuchung des Sprachverhaltens von Migranten gibt es neben den Untersuchungen zur Sprachform (Gastarbeiterdeutsch, petit nègre usw.) eine ausgeprägte Tendenz zur Analyse des situativen Sprachverhaltens und hier naturgemäß des CodeWechsels. Auch hier tritt das Problem der Vielfältigkeit der beteiligten Sprachen auf, d. h. das Verhältnis zwischen Standardsprache und Dialekt sowohl in der Herkunftssprache als auch in der Umgebungssprache (vgl. u. a. Lüdi et al. 1984). Die Untersuchungen des situativen Sprachverhaltens geben auch hier entscheidende Hinweise auf die Sprachbewertungen und - durch die Art der Sprachwechsel im Verhältnis zur grammatischen Struktur der Äußerungen - auf die Entwicklung von spezifischen Kompetenzformen des zweisprachigen Sprechers.

Ein weiterer wichtiger Untersuchungsgegenstand ist, wie sich die Spracheinschätzung und das Sprachbewußtsein bei Migranten entwickelt in Abhängigkeit von den Kontakten mit der Herkunftsgemeinschaft und der sozialen und kulturellen Organisation im Gastland (häufig auch Einwanderungsland). Billiez (1986) zeigt hier z. B. signifikante Unterschiede zwischen algerischen und spanischen Migranten in Grenoble, wobei die Spanier tendenziell ihre Sprache als praktizierte Sprache ihrer eigenen kulturellen Welt beibehalten, während für die Algerier das Arabische zunehmend einen nicht mehr praktischen, sondern nur noch ideellen Status bekommt. Auch für die türkischen Gastarbeiter bzw. Migranten in der BRD sind diese Vorgänge der wachsenden Distanz von der Heimatkultur und -sprache, die Versuche der kulturellen Fixierung auch der zweiten Generation 
durch die Elterngeneration und die sich daraus ergebenden Spannungen Gegenstand der Forschung (vgl. z. B. die Beiträge zum Thema in Bausch 1982).

\subsection{Die Binnengliederung der Stadt}

Eine mehr oder weniger dauerhafte sozialgeographische Gliederung in Stadtviertel oder vergleichbare Areale bis hin zu Existenz von „Orten“ innerhalb der Stadt gehört zur normalen Struktur der Stadt. Als Faktoren der Entwicklung von relativ eigenständigen städtischen Einheiten spielen u.a. eine Rolle:

- der Fortbestand älterer Strukturen (dörflicher, kleinstädtischer) in einem neuen städtischen Kontext,

- Stadterweiterung und Umgestaltung von Stadtvierteln; wobei es für bestimmte Areale offensichtlich typische Entwicklungen gibt (z.B. bestimmte Innenstadtviertel, Sanierungsgebiete, aus Wohnvierteln hervorgegangene Geschäftsviertel).

- die gruppenweise Migration, bei der die früheren Lebensweisen in die neue Umgebung verpflanzt werden (z. B. als ein importiertes „Dorf in der Stadt“).

Beim Erhalt bestehender kleinräumiger Organisationsformen wie auch bei deren Umgestaltung oder bei der Neubildung von städtischen Teileinheiten spielen Prozesse der Konstitutierung relativ überschaubarer Lebensbereiche im Rahmen großer sozialer Komplexe eine Rolle. Dieser Prozeß kann mit einer relativ großen Bezogenheit auf den Lebenskreis des Stadtteils verbunden sein mit der Folge einer ..Provinzialität“ der Bewohner, wie sie z. B. von der amerikanischen Stadtsoziologie der sog. Chicago Schule für bestimmte Stadtbezirke festgestellt wurde (vgl. z. B. Suttles über die .Adams Area“ in Chicago, 1968). Die Bedeutung des Wohnumfeldes wird in der soziologischen Wohnquartierforschung hervorgehoben. Erhalt und Wiederbelebung kleinräumiger Strukturen in der Stadt sind auch für die Volkskunde/Kulturanthropologie ein wichtiges Thema (vgl. z. B. Kohlmann/Bausinger 1985, 9; vgl. auch Ruppert 1985 über „Stadtteilkultur“).

Die Einteilung der Stadt nach Stadtteilen bzw. Wohnquartieren und städtischen Arealen wird in der soziolinguistischen Stadtforschung vielfach als Auswahl- und Einordnungsrahmen und für innerstädtische Kontrastbildungen benutzt (z. B. auch im Berliner Projekt mit der Gegenüberstellung von einem Arbeiter- und einem Mittelschichtstadtteil; vgl. Dittmar/Schlobinski 1987). Darüber hinaus werden Stadtteile auch in ihrer Orientierungs- und Abgrenzungsfunktion für die Bevölkerung untersucht. ${ }^{7}$ Besonders wichtig ist hier die schon klassische Untersuchung von Milroy (1980) zu zwei Stadtteilen von Belfast, in der sprachliche Resistenz gegen eine sprachlich und ökonomisch dominante Umgebung mit der Geschlossenheit der städtischen Milieus erklärt wird.

Das Stadtviertel oder vergleichbare relativ stabile sozialräumliche Gliederungen der Stadt sind offensichtlich ein guter Ansatzpunkt für die Behandlung der Fragestellung, wie sich der Prozeß der sozialen Organisation in Form von lokal begrenzten Ortsstrukturen im Rahmen der Stadt einerseits und die soziale Segregation und damit verbundene Orientierungen auf überlokale Strukturen andererseits zueinander verhalten. ${ }^{8}$ Stadtvier-

7 So in italienischen Untersuchungen, vgl. Sobrero 1978, auch in Untersuchungen zu Barcelona.

$8 \mathrm{Vgl}$. auch Mattheiers Annahme über die mögliche Auflösung der Stadt in verschiedene Ortsgemeinschaften (1985b). 
teluntersuchungen ergeben auch bei Untersuchungen zur Großstadt vielleicht den unmittelbarsten Bezug zu der wesentlich im Rahmen der Ortsforschung geführten Diskussion um die Vorstellung von der Ortsgemeinschaft als Sprachgemeinschaft (vgl. z. B. die Zusammenfassungen in Dittmar/Schlobinski 1985 und Wiesinger 1985). Dabei wird die Tragfähigkeit des Konzepts in Frage gestellt, bis hin zur Empfehlung, auf das Konzept ganz zu verzichten (vgl. u. a. Romaine 1982, 43). Die Befunde laufen darauf hinaus, daß schon kleine Gemeinschaften sprachlich nicht homogen sind und daß soziale Integration auch auf der Grundlage sprachlicher Heterogenität möglich ist. Die Ortsforschung hat wesentliche Impulse geliefert für die Beobachtung der sozialen Integration. Ein Beispiel gibt die Studie von Dorian (1982), die anhand einer gälisch-englischen Gemeinschaft die Bedingungen für Integration in die lokale, gälische Gemeinschaft untersucht. Dabei stellt sich heraus, daß bei hinreichender passiver Sprachkompetenz und ausgebildeter sozialer/pragmatischer Kompetenz eine Zugehörigkeit zur lokalen Gemeinschaft ohne weiteres möglich ist.

Diese Diskussion weist eine gewisse Analogie zu der um die Varietäten bzw. Sprachschichten auf. Auch bei der Frage der Gemeinschaft gibt es die Tendenz, ein globales, nicht befriedigend zu definierendes Konzept zugunsten eines Beschreibungsbegriffes wie ,Netzwerk' aufzugeben. Aber es bleibt wiederum das Problem, daß übergreifende soziale Einheiten wie ,Gemeinschaften" oder ,soziale Welten" als Orientierungs- und Aktivitätsrahmen für die Gesellschaftsmitglieder von Bedeutung sind, daß jedoch diese Rahmen mit Hilfe eines Konzeptes wie ,Netzwerk' nicht zufriedenstellend zu rekonstruieren sind.

Für die weitere Bearbeitung des Problems scheint es angemessen zu sein, sich auf einen variablen Zusammenhang zwischen Sprache und Gemeinschaftsstruktur einzustellen, der daher nicht definitorisch festgesetzt werden kann, sondern gerade Untersuchungsgegenstand sein muß. Dabei kommt es voraussichtlich darauf an, einerseits die Eigenschaften des sprachlichen Verhaltens umfassender als bisher üblich zu erfassen, und andererseits auch die Herstellung von sozialem Zusammenhalt aspektreicher zu untersuchen, als es z. B. in einer reinen Netzwerkanalyse geschieht. Zwei Punkte scheinen mir dabei wichtig. Zum einen muß der Zusammenhang von sozialer Organisation und Kommunikationsstrukturen umfassender analysiert werden (also sozialökologische und sozialstrukturelle Formen wie Schauplätze, Situationstypen, formale Organisationen und Institutionen in ihrem Verhältnis zu Chancen und Zwängen der Kommunikation) (vgl. u. a. Kallmeyer/Keim/Nikitopoulos 1982). Schwierigkeiten bereiten hier die vermittelnden Strukturkonzepte auf „Mezzo-Ebene“ zwischen Gruppen und direkt beobachtbaren Interaktionsereignissen einerseits und andererseits den globalen, durch formale Organisation bestimmten Einheiten. Zum anderen ist parallel zur Untersuchung der Sprachwahrnehmung und des Sprachwissens auch die Untersuchung der Wahrnehmung von und des Wissens über soziale Strukturen wichtig. Komplexe Gemeinschaften werden wesentlich auch über das Reden über die anderen (in direkter Kommunikation und medial vermittelt) und über die Ausbildung sozialer Repräsentationen von ihnen hergestellt. Solche Arten der vermittelten Präsenz können ansonsten disparate Welten verketten (zusammen mit einem administrativen oder organisatorischen Rahmen). 


\subsection{Gruppen, Milieus, soziale Subkulturen}

Es gibt eine Reihe schon klassischer Gruppen- und Milieuuntersuchungen unterschiedlicher Ausrichtung. Dazu gehören Studien aus der symbolischen Anthropologie, der Soziologie und der Soziolinguistik, z. B. zur „taxi dance hall“ von Cressey (1932), über die „Street corner society“ von Whyte (1955), zu den schwarzen Jugendlichen in New York von Labov (1972) oder auch die Studien von Jugendkulturen in England (vgl. Willis 1978). Es handelt sich hier um Gruppen und Milieus, die in ihrer Existenz an die Stadt gebunden sind, sie sind typisch städtische Erscheinungen; sie sind in ihrer Zusammensetzung, den Formen ihrer sozialen Organisation und ihrem sozialräumlichen Verhalten städtisch. Insofern sind sie ein gutes Beobachtungsobjekt für die detaillierte Untersuchung des Sprachverhaltens in der Stadt (vgl. auch Dittmar/Schlieben-Lange 1982 b, 82).

Eine wichtige Aufgabe der Gruppenforschung im Rahmen der Soziolinguistik der Stadt ist es zu untersuchen, in welcher Weise derartige Gruppen, Milieus und Subwelten im städtischen Raum einerseits ggf. von überlokalen, in der Regel überregionalen, auch übernationalen Bezügen geprägt sind wie die verschiedenen Spielarten der Jugendkultur, andererseits aber auf das spezifische städtische Umfeld bezogen sind. Es kommt darauf an zu zeigen, welche Rolle die Gruppe für die städtische Umwelt spielt. In diese Richtung gehen u. a. neuere Studien zu Gruppen von Jugendlichen, wie z. B. die Arbeit von Laks (1980). Im theoretischen Rahmen der Soziologie des sprachlichen Marktes und der Ausbildung sozialer Unterschiede von Bourdieu untersucht Laks mit Hilfe einer Kombination von Ethnographie und Labovscher Variablenanalyse eine Gruppe von Jugendlichen in einer Stadt der Pariser Banlieue, Villejuif, die sich in einem Jugendzentrum angesiedelt haben und die in ihren Gruppeninteraktionen sprachliche Unterschiede reproduzieren, die aufgrund der sozialen Zusammensetzung der Stadt im Rahmen der städtischen Gesellschaft relevant sind; es gibt hier also eine Korrespondenz von gruppeninternem und externem sprachlichem Markt, und die Prozesse der Durchsetzung der dominanten Sprachnorm, die in der städtischen Gesellschaft ablaufen, vollziehen sich auch innerhalb der Gruppe. ${ }^{9}$

Gerade Untersuchungen von Gruppen oder auf bestimmten Schauplätzen angesiedelten städtischen Kulturen geben aufgrund der Begrenztheit des Gegenstandes die Möglichkeit zu einer sehr detaillierten Beschreibung des sprachlichen Verhaltens. In einem solchen begrenzten Rahmen ist am ehesten auch eine linguistisch aspektreiche Untersuchung auf mehreren Sprachebenen und die Zuordnung der Einzelbefunde zu kulturell geprägten Mustern des sprachlichen Verhaltens im Sinne einer Soziostilistik möglich.

9 Die Binnendifferenzierung von Gruppen spielt auch in Rebaudière-Paty 1985 eine Rolle. Gruppen und ihre Einordnung in übergreifende Strukturen sind ein wichtiger Untersuchungsgegenstand im Mannheimer Projekt und auch in der anlaufenden Untersuchung von G. Klein in Neapel (Klein 1987). Ähnlich gute Chancen für die Beobachtung von lokalen Milieus bzw. Subwelten und für die differenzierte Untersuchung der Reproduktion und Produktion städtischen Sprachverhaltens im Rahmen kleinräumiger sozialer Organisation bieten Vereine (entsprechende Untersuchungen laufen u.a. im Berliner und Mannheimer Projekt). 


\subsection{Die Stadt als Raum interkultureller Kommunikation}

Die Forschung zur interkulturellen Kommunikation wird häufig nicht explizit auf sozialräumliche Einheiten wie die Stadt bezogen, faktisch ist dieser Bezug aber in den meisten Fällen gegeben, und er läßt sich auch systematisch herstellen (vgl. z. B. Gumperz 1984). Aufgrund der Migration und der Gemeinschaftsbildungsprozesse innerhalb der Stadt ist die Stadt ein Raum, in dem interkulturelle Kontakte gleichsam normal sind. Der interkulturelle Kontakt erscheint dabei als ein besonders markanter Fall des Kontakts von Fremden im offenen städtischen Territorium oder in institutionellen Kontexten; dabei gelten gerade alle Voraussetzungen an geteiltem Wissen auf der Grundlage von Bekanntheit oder zumindest Vertrautheit mit der sozialen Kategorie des anderen nicht, wie sie in den Gruppen- und Milieuuntersuchungen vor allem hervortreten.

Die Anonymität in der Stadt ist ein altes Thema der soziologischen Stadtforschung aus den 20er Jahren. Gerade als Ergänzung zur Erforschung kleinräumiger sozialer Strukturen in der Stadt ist dieser Aspekt wichtig. Die Markierung von Vertrautheit und Fremdheit bzw. von eigener und fremder Welt durch Sprachwahl ist auch ein fester Bestandteil von Untersuchungen zum code-switching (vgl. u.a. Gumperz 1982a). Darüber hinaus kann man anknüpfen an Überlegungen über die Bedeutung der Fremdwahrnehmung und Selbstdarstellung gegenüber Fremden und die davon abgeleitete Bedeutung von einfachen sprachlichen Merkmalen als soziale Indikatoren (vgl. u. a. die Unterscheidung von Indikatoren, Markern und Stereotypen bei Labov). Die interkulturelle Kommunikation stellt eine Zuspitzung der Fremdheitssituation dar, insofern hier auch der Rekurs auf kulturelles Hintergrundwissen als Verständigungsgrundlage fragwürdig wird.

\subsection{Sprachgeschichte der Stadt}

Dittmar/Schlieben-Lange (1982b) heben die besondere Entwicklung der historischen Stadtsprachenuntersuchungen im romanischen Sprachraum hervor (u.a. Sobrero 1978) und konstatieren ansonsten: ,,besonders vernachlässigt wurde die Sprachgeschichte der Städte“ (Dittmar/Schlieben-Lange 1982b, 66); Brang (1986, 66) stellt etwas Ähnliches für den slavischen Sprachraum fest, trotz der frühen programmatischen Formulierungen von Vinogradov zur Geschichte der Stadtsprache von Moskau und Petersburg und trotz der Arbeiten von Wieczorkiewicz zu Warschau (Angaben bei Brang 1986). In der Germanistik sind seitdem eine Reihe von Projekten zur historischen Stadtsprachenforschung hervorgetreten: Cherubim/Flechsig (1984) zu Braunschweig, Maas (1987) zu Osnabrück, Hoffmann/Mattheier (1985) zu Köln und Wildgen (1986) zu Bremen. Zumindest aus deutscher Perspektive sieht es so aus, als würde das von Dittmar/Schlieben-Lange formulierte Defizit allmählich ausgeglichen.

Interessant an den neueren historischen Arbeiten ist, daß Gesichtspunkte aus der Gemeindeforschung und der ethnographischen Soziolinguistik auf die Untersuchung historischer Verhältnisse übertragen wird. So weisen Hoffmann/Mattheier darauf hin, daß die historische Stadtsprachenforschung einen weiten Begriff von „Stadtsprache“ zugrunde zu legen habe, wie er sich gerade in der Erforschung gegenwärtiger Verhältnisse in der Orts- und Stadtsprachenforschung ergeben hat: ,es reicht nicht aus, die Wechselwirkung 
zwischen Stadt und Sprache auf den Aspekt der Entstehung der Einheitssprache zu beschränken“ (S. 1837), wenn bei dieser Entwicklung dieser Einheitssprache die Städte natürlich auch eine leitende Rolle gespielt haben, sondern es geht um die Stadtsprache als „das Ensemble aller zu einer Zeit aktiv verwendeten und miteinander verwandten Varietäten“ sowie zusätzlich um ,alle Sprachen, die in früheren Zeiten innerhalb der Stadt schriftlich und mündlich verwendet und verstanden wurden" (S. 1838). Interessanterweise findet z. T. auch eine Annäherung an die Analyse der konkreten Verwendungszusammenhänge und der situativen Konstitution von Äußerungen trotz aller damit verbundenen Probleme statt. So versucht z. B. Maas (1987) in der Untersuchung der Verdrängung des Niederdeutschen durch das Oberdeutsche im Osnabrück der frühen Neuzeit Texte als Inskriptionen einer Schreibpraxis zu analysieren und über die Korrekturen, Reformulierungen usw. sprachliche Orientierungen der Schreiber zu erfassen.

Angesichts der verstärkten historischen Forschung bleibt vielleicht zu wünschen, daß noch stärker im Austausch mit der Gegenwartsforschung auf gemeinsame theoretische Rahmenvorstellungen für sprachliche und soziale Konstellationen und Prozesse hingearbeitet wird, und daß in den aktuellen Untersuchungen auch das historische Bewußtsein als eine wichtige Komponente der Identitätsbildung behandelt wird. In allen Stadtsprachenprojekten wird im Prinzip eine historische Einordnung vorgenommen. Darüber hinaus ist es aber sicher interessant, die Wahrnehmung der Spuren historischer Prozesse in der Gegenwart, die Konstruktion der eigenen Geschichte und die Herausarbeitung von Identifikationsobjekten zu verfolgen (u. a. als Elemente einer „städtischen Mythologie" aus besonderen, lokal orientierten Figuren - also neben historisch bedeutsamen Figuren wie Zille in Berlin Figuren wie der „Blumenpeter“ in Mannheim, das „Gänseliesel“ usw.). Sicher gewinnen in den nächsten Jahren auch Ansätze zur Erfassung der Sprachbiographie als einem wichtigen Glied in der Untersuchung von Wandlungsprozessen größere Bedeutung.

\section{Bibliographie}

Allardt, Erik/Miemois, Karl Johan/Starck, Christian (1979): ..Multiple and varying criteria for membership in a linguistic minority. The case of the Swedish speaking minority in metropolitan Helsinki“, in: Research group for sociology, University of Helsinki, Research Reports No. 21.

Auer, Peter (1984): „On the meaning of conversational code-switching“, in: Auer/di Luzio 1984. S. $87-108$.

Auer, Peter/di Luzio, Aldo (Hrsg.) (1984): Interpretative sociolinguistics. Migrants - children migrant children. Tübingen 1984.

Auer, Peter/di Luzio, Aldo (Hrsg.) (1987): Dialect, variation and convergence. Berlin 1987

Auer, Peter/Mössle, Angela (1986): „A case of convergence and its interpretation: MHG î and $\hat{u}$ in the city dialect of Constance", in: Auer/di Luzio 1986

Bausch, Karl-Heinz (Hrsg.) (1982): Mehrsprachigkeit in der Stadtregion. Jahrbuch 1981 des Instituts für deutsche Sprache. Düsseldorf 1982 (Sprache der Gegenwart 56).

Beatens Beardsmore, Hugo (1983): „The ,supreme-language hypothesis applied to Brussels". II Nelde, Peter Hans (Hrsg.) Gegenwärtige Tendenzen der Kontaktlinguistik. Bonn 1983, S. 15-29 (Plurilingua I).

Besch, Werner/Hufschmidt, Jochen/Kall-Holland, Angelika/Klein, Eva/Mattheier, Klaus J. (1981): Sprachverhalten in ländlichen Gemeinden. Ansätze zur Theorie und Methode. Forschungsbericht Erp-Projekt. Band 1. Berlin [Band 2 siehe Hufschmid et al.]. 
Besch, Werner/Mattheier, Klaus (Hrsg.) (1985): Ortssprachenforschung. Beiträge zu einem Bonner Kolloquium. Berlin 1985.

Besch, Werner/Knoop, Ulrich/Puschke, W./Wiegand, Herbert E. (Hrsg.) (1983): Dialektologie. Ein Handbuch zur deutschen und allgemeinen Dialektforschung. Berlin 1983.

Blom, Jan Peter/Gumperz, John J. (1972): ..Social meaning in linguistic structure: Code switching in Norway", in: Gumperz/Hymes 1972, S. 407-434.

Bodzenta, Erich/Speiser I./Thum, K. (1981): Wo sind Großstädter daheim? Studien über Bindungen an das Wohnviertel. Wien 1981.

Bourdieu, Pierre (1982): Ce que parler veut dire. L’économie des échanges linguistiques. Paris 1982.

Bourdieu, Pierre (1984): „Capital et marché linguistique“, in: Linguistische Berichte 90, 1984. S. 3-24.

Brang, Peter (1986): „Zum gegenwärtigen Stand der slawischen Stadtsprachenforschung“, in: Zeitschrift für Slawische Philologie 46, 1986, S. 140-169.

Bräutigam, Kurt (1934): Die Mannheimer Mundart. Heidelberg 1934.

Brekle, Herbert E./Maas, Utz (Hrsg.) (1986): Sprachwissenschaft und Volkskunde. Perspektiven einer kulturanalytischen Sprachbetrachtung. Opladen 1986.

Brinkmann to Broxten, Eva (1986): Stadtsprache-Stadtmundart. Studie zum Gebrauch und zur Funktion mundartnaher Sprachvarietäten in Frankfurt/Main. Tübingen 1986.

Burgess, E. W. (1929): Urban areas of Chicago: An experiment in social science research. Chicago 1929.

Chauvin, Marcel (1985): „Transformation d'une forme regionale de Français en une variété sociale urbaine?", in: International journal of the sociology of language 54, 1985, S. 5-15.

Cherubim, Dieter/Flechsig, W. (1984): „.Stadtsprachenforschung - am Beispiel Braunschweigs“, in: Mitteilungen der Technischen Universität Carolo-Wilhelmina zu Braunschweig 19, 1984. S. 56-62.

Cressey, Paul G. (1932/1969): The taxi dance hall. A sociological study in commercialized recreation and city life. Chicago 1932. Wieder: Montclair, N. J. 1969.

Deprez, Kas/Persoons, Yves (1983): „Mehrsprachigkeit und Großstadt. Aspekte der ethnolinguistischen Identität der flämischen Gemeinschaft in Brüssel“, in: Nelde, Peter Hans (Hrsg.), Mehrsprachigkeit/Multilingualism. Bonn 1983, S. 77-94 (Plurilingua IV).

Debus, Friedhelm (1962): „Zwischen Mundart und Hochsprache. Ein Beitrag zur Stadtsprache Stadtmundart und Umgangssprache“" in: Zeitschrift für Mundartforschung 29, 1962, S. 1-43.

Debus, Friedhelm (1978): „Stadt-Land-Beziehungen in der Sprachforschung. Theoretische Ansätze und Ergebnisse", in: Wiegelmann, G. (Hrsg.), Kulturelle Stadt-Land-Beziehung in der Neuzeit. Münster 1978, S. 115-158.

Denison, N. et al. (1977): „Linguistic Behaviour in Graz - a preliminary report “, in: Innsbrucker Beiträge zur Sprachwissenschaft.

Dittmar, Norbert/Schlieben-Lange, Brigitte (Hrsg.) (1982a): Die Soziolinguistik in den romanischsprachigen Ländern. La sociolinguistique dans les pays de langue romane. Tübingen 1982.

Dittmar, Norbert/Schlieben-Lange, Brigitte (1982b): „Stadtsprache. Forschungsrichtungen und Perspektiven einer vernachlässigten soziolinguistischen Disziplin“" in: Bausch 1982, S. 9-86.

Dittmar, Norbert/Schlobinski, Peter (1985): „Die Bedeutung von sozialen Netzwerken für die Erforschung von Ortssprachen“, in: Besch/Mattheier 1985, S. 158-188.

Dittmar, Norbert/Schlobinski, Peter (Hrsg.) (1987): The Sociolinguistics of Urban Vernaculars. Case studies and their evaluation Berlin 1987.

Dittmar, Norbert/Schlieben-Lange, Brigitte/Schlobinski, Peter (1982): „Teilkommentierte Bibliographie zur Soziolinguistik von Stadtsprachen", in: Bausch 1982, S. 391-423.

Dittmar, Norbert/Schlobinski, Peter/Wachs, Inge (1986): Berlinisch. Studien zum Lexikon, zur Spracheinstellung und zum Stilrepertoire. Berlin 1986.

Donath, Joachim/Pape, Ruth/Roloff, Marion/Schönfeld, Helmut (1981): „Beschreibung einer empirischen Untersuchung zur Sprachvarianz", in: Hartung, Wolfdietrich/Schönfeld, Helmut et al. (Hrsg.), Kommunikation und Sprachvariation. Berlin 1981, S. 308-440 (Reihe Sprache und Gesellschaft Bd. 17). 
Dorian, Nancy C. (1982): „Defining the speech community to include its working margins“, in: Romaine 1982, S. 25-33.

Dressler, Wolfgang U./Wodak, Ruth (1982): „Sociophonological methods in the study of sociolinguistic variation in Viennese German", in: Language in society 11, 1982, S. 339-370.

Dyhr, Mogens/Zinnt, Ingeborg (1985): „Vorüberlegungen zu einem Projekt ,Sprachvariation in Flensburg،", in: Kopenhagener Beiträge zur Germanistischen Linguistik 23, 1985, S. 91-104.

Friebertshäuser, Hans/Dingeldein, Heinrich J. (1985): „Wortgeographie der städtischen Alltagsprache. Ein Forschungsprojekt zum aktuellen Sprachgebrauch in Hessen", in: Zeitschrift für Dialektologie und Linguistik 52, H. 1, 1985, S. 43-52.

Friedrichs, Jürgen (1977): Stadtanalyse. Soziale und räumliche Organisation der Gesellschaft. Reinbek 1977.

Froitzheim, Claudia (1984): Artikulationsnormen der Umgangssprache in Köln. Tübingen.

Gal, Susan (1979): Social determinants of linguistic change in bilingual Austria. New York 1979.

Gans, Herbert, J. (1962): The urban villagers. Group and class in the life of Italian-Americans Glencoe 1962

Gardener-Chloros, Penélopé (1985): „Language selection and switching among Strasbourg shoppers", in: International journal of the sociology of language 54, 1985, S. 115-134

Gauchat, Louis (1905): „l'unité phonétique dans le patois d'une commune“, in: Aus Romanischen Sprachbändchen und Literaturen - Festschrift Heinrich Morph. Halle, S. 175-232.

Gerndt, Helge (1985): „Großstadtvolkskunde - Möglichkeiten und Probleme“, in: Kohlmann Bausinger 1985 , S. $11-19$.

Graumann, Carl F./Schneider, Gerhard (1982): Städtische Umwelt: Identität und Identifikation. München

Greverus, Ina-Maria (1978): Kultur und Alltagswelt. Eine Einführung in Fragen der Kulturanthropologie. München 1978.

Gubert, Renzo (1978): La cittá bilingue. Bolzano.

Gumperz, John J. (1972): „The speech community“, in: Giglioli, P. P. (Hrsg.): Language and social context. Harmondsworth 1972, S. 219-231.

Gumperz, John J., (1981): Language in social groups. Stanford, CA 1981.

Gumperz, John J. (1982a): Discourse strategies. Cambridge 1982 (Studies in interactional sociolinguistics 1).

Gumperz, John J. (Hrsg.) (1982b): Language and social identity. Cambridge 1982 (Studies in interactional sociolinguistics 2).

Gumperz, John J. (1984): „Ethnography in urban communication“, in: Auer/di Luzio 1984. S. $1-12$.

Gumperz, John, J./Hymes, Dell H. (Hrsg.) (1964): „The ethnography of communication“, in: American antropologist,'special publication, vol. 66,6; part II, 1964.

Gumperz, John J./Hymes, Dell H. (Hrsg.) (1972): Directions in social linguistics. The ethnography of communication. New York 1972.

Haugen, Einar (1972): „The ecology of language“, in: Haugen, Einar, The ecology of language. Essays, selected and introduced by Anwar S. Dill. Stanford, Cal. 1972, S. 325-339.

Hermann, E. (1929): „Lautveränderungen in den Individualsprachen einer Mundart“, in: Nachrichten der Gesellschaft der Wissenschaften zu Göttingen, Philosophisch-historische Klasse II. S. $195-214$.

Hoffmann, Walter/Macha, Jürgen (1985): „Zusammenfassung der Diskussionen des Ortssprachenkolloquiums", in: Besch/Mattheier (1985) S. 283-289.

Hoffmann, Walter/Mattheier, Klaus (1985): „Stadt und Sprache in der neueren deutschen Sprachgeschichte: eine Pilotstudie am Beispiel von Köln“, in: Besch, Werner/Reichmann, Oskar/Sonderegger, Stefan (Hrsg.), Sprachgeschichte. Ein Handbuch zur Geschichte der deutschen Sprache und ihrer Erforschung. Zweiter Halbband, Berlin 1985, S. 1837-1865.

Hufschmidt, Jochen/Klein, Eva/Mattheier, Klaus J./Mickartz, Heinrich (1983): Sprachverhalten in ländlichen Gemeinden. Dialekt und Standardsprache im Sprecherurteil. Forschungsbericht ErpProjekt. Band 2. Berlin [Band 1 siehe Besch et al.]. 
Hugger, Paul (1985): „Von der ,Dorfidylle' zum Alltag eines Industriequartiers. Die Entwicklung der Basler Bannmeile Kleinhüningen", in: Kohlmann/Bausinger 1985, S. 63-75.

Hymes, Dell H. (1977): Foundation in sociolinguistics. An ethnographic approach. London 1977. Dt.: Soziolinguistik. Zur Ethnographie der Kommunikation. Frankfurt 1979.

Kallmeyer, Werner (Hrsg.) (i.V.): Kommunikation in der Stadt - exemplarische Analysen des Sprachverhaltens in Mannheim. Berlin i. V.

Kallmeyer, Werner/Keim, Inken/ Nikitopoulos, Pantelis (1982): ..Zum Projekt .Kommunikation in der Stadt "“, in: Bausch 1982, S. 345-390.

Klein, Gabriella (Hrsg.) (1987): Sociolinguistica urbana in Italia e Europa occidentale. Voraussichtlich Lecce 1987.

Kohlmann, Theodor/Bausinger, Hermann (Hrsg.) (1985): Großstadt. Aspekte empirischer Kulturforschung. 24. Deutscher Volkskunde-Kongreß in Berlin vom 26.-30. September 1983. Berlin 1985.

Kolde, Gottfried (1981): Sprachkontakte in gemischtsprachigen Städten. Vergleichende Untersuchungen über Voraussetzungen und Formen sprachlicher Interaktion verschiedensprachiger Jugendlicher in den Schweizer Städten Biel/Bienne und Fribourg/Freiburg. Wiesbaden 1981 (Ztft. für Dialektologie und Linguistik, Beiheft 37).

Labov, William (1966): The social stratification of English in New York city. Washington D. C.. 1966.

Labov, William (1972): Language in the inner city. Philadelphia 1972.

Laks, Bernard (1980): Différenciation sociale: quelques problèmes de sociolinguistique française. Thèse de 3ème cycle de linguistique, Paris 1980.

Lüdi, Georges/Py, Bernard (1982): „Approches linguistiques des migrations internes et externes“. in: Grazer Linguistische Studien 16, 1982, S. 89-104.

Lüdi, Georges/Oesch-Serra, Cecilia/Py, Bernard (1984): ..Proposals for a heuristic model of bilingualism for various groups of migrants", in: Auer/di Luzio 1984, S. 13-27.

Maas, Utz (1986): „Volkskundliches (Kultur) in der Sprachwissenschaft“, in: Brekle. Herbert, E./ Maas, Utz (Hrsg.): Sprachwissenschaft und Volkskunde. Perspektiven einer kulturanalytischen Sprachbetrachtung. Opladen 1986, S. 33-69.

Maas, Utz (1987): „Die sprachlichen Verhältnisse in Osnabrück zu Beginn des 17. Jahrhunderts“. in: Auer/di Luzio 1987.

Mattheier, Klaus J. (1982): „Sprachgebrauch und Urbanisierung. Sprachveränderungen in kleinen Gemeinden im Umfeld großer Städte", in Bausch 1982, S. 87-107.

Mattheier, Klaus J. (1983): ..Mehrsprachigkeit und Ortssprachenforschung. Einige theoretische und methodische Probleme der Ortssprachenforschung in Mehrsprachen- und Mehrvarietäten-Ortsgemeinschaften", in: Nelde, Peter Hans (Hrsg.), Mehrsprachigkeit/Multilingualism. Bonn 1983. S. $163-176$.

Mattheier, Klaus J. (1985a): „Ortsloyalität als Steuerungsfaktor von Sprachgebrauch in örtlichen Sprachgemeinschaften", in: Besch/Mattheier 1985, S. 139-157.

Mattheier, Klaus J. (1985b): „Ortssprachenanalyse. Allgemeine Überlegungen und Vorschläge“. in: Kopenhagener Beiträge zur Germanistischen Linguistik 23, 1985, S. 7-23.

Mattheier, Klaus J. (1979): Sprachvariation und Sprachwandel. Untersuchungen zur Struktur und Entwicklung von Interferenzprozessen zwischen Dialekt und Hochsprache in einer ländlichen Sprachgemeinschaft des Rheinlandes. Habil.-Schr. Bonn 1979 ms.

Mattheier, Klaus, J./Besch, Werner (1985): ..Ortssprachenforschung. Einleitende Überlegungen“, in: Besch/Mattheier 1985, S. 9-23.

Mihm, Arend (Hrsg.) (1985): Sprache an Rhein und Ruhr. Dialektologische und soziolinguistische Studien zur sprachlichen Situation im Rhein-Ruhr-Gebiet und ihrer Geschichte. Stuttgart 1985 (Zeitschrift für Dialektologie und Linguistik, Beihefte, Heft 50).

Mihm, Arend (1985c): „Sprachlandschaft Duisburg“, in: Mihm (1985a), S. 201-222.

Milroy, Lesly (1980): Language and social networks. Oxford 1980.

Mitzka, Walther (1928): Sprachausgleich in den deutschen Mundarten bei Danzig. Königsberg 1928. 
Munske, H. H. (1983): „Umgangssprache als Sprachkontakterscheinung“, in: Besch/Knoop/Puschke/Wiegand 1983, Bd. 2, S. 1002-1017.

Nelde, Peter Hans (1978): „Sprachkonflikt und Sprachwechsel in Brüssel“, in: Ureland, P. S. (Hrsg.), Sprachkontakt im Nordseegebiet. Akten des 1. Symposiums über Sprachkontakt in Europa, Mannheim 1977. Tübingen 1978, S. 19-41.

Pedersen, Inge Lise (1985): „Urbanisierung und Sprachvariation. Bericht über eine laufende Ortssprachenuntersuchung auf Fünen", in: Kopenhagener Beiträge zur Germanistischen Linguistik 23 (1985), S. $105-116$.

Radtke, Ingulf (1972): ..Soziolinguistik von Stadtsprachen. Tendenzen soziolinguistischer Forschungen in der BRD", in: Germanistische Linguistik 3, 1972, S. 441-517.

Raith, Joachim (1985): „Der Terminus ,Sprachgemeinschaft als soziolinguistisches Konzept in der Mehrsprachigkeitsforschung", in: Nelde, Peter Hans (Hrsg.), Gegenwärtige Tendenzen in der Kontaktlinguistik. Bonn 1983, S. 41-51 (Plurilingua I).

Rebaudière-Paty, Madeleine (1985): „Langues et Identités dans le bassin houiller Lorraine: Une approche de terrain", in: International journal of the sociology of language 1985, S. 17-40.

Romaine, Suzanne (1982): Sociolinguistic variation in speech communities, London 1982.

Romaine, Suzanne (1982a): „What is a speech community?”, in: Romaine 1982, S. 13-24.

Rosenberg, Klaus-Peter (1986): „Der Berliner Dialekt und seine Folgen für die Schüler. Geschichte und Gegenwart der Stadtsprache Berlins sowie eine empirische Untersuchung der Schulprobleme dialektsprechender Berliner Schüler. Tübingen 1986.

Rupert, Wolfgang (1985): „Was ist Stadtteilkultur?“, in: Kohlmann/Bausinger 1985, S. 77-83.

Schenk, Michael (1983): „Das Konzept des sozialen Netzwerkes“, in: Neidhart, Friedhelm (Hrsg.): Gruppensoziologie. Perspektiven und Materialien. Zeitschrift für Soziologie und Sozialpsychologie, Sonderheft 25 (1983) S. 88-104.

Schenk, Michael (1984): Soziale Netzwerke und Kommunikation. Tübingen.

Senft, Gunter (1982): Sprachliche Varietät und Variation im Sprachverhalten Kaiserslauterer Metallarbeiter. Untersuchungen zu ihrer Begrenzung, Beschreibung und Bewertung. Bern/Frankfurt 1982 .

Sobrero, Alberto A. (1978): „Borgo, città, territorio: alcuni problemi di metodo nella dialettologia urbana", in: Rivista italiana di dialettologia 2, 1978, S. 9-21.

Sobrero, Alberto A. (1985): „Il projetto NADIR (Nuovo Atlante dei Dialetti e dell' Italiano per Regioni)", in: Atti del XVII Congresso Internazionale di Linguistica e Filologia Romanza 1985.

Sobrero, Alberto A. (1987): „Conversando, fra dialetto e lingua. Microconvergenze“, in: Auer/di Luzio 1987.

Sornig, Karl (1977): „Bemerkungen und Überlegungen zur Bearbeitung der Grazer Protokolle“, in: Grazer Linguistische Studien 6, 1977, 9-57.

Sornig, Karl (1978): „Zwischenbericht von der Arbeit an den Grazer Protokollen“, in: Grazer Linguistische Studien 8, 1978, S. 136-162.

Suttles, Gerald (1968): The social order of the slum. Ethnicity and territory in the inner city. Chicago 1968.

Suttles, Gerald (1972): The social construction of communities. Chicago/London 1972.

Tabouret-Keller, Andrée (1985): „Introduction“, in: International journal of the sociology of language 54, 1985, S. 5- 15.

Thies, Udo (1982): „Sprachvariationen im Ruhrgebiet - ein Beitrag zur Methodologie stadtsprachlicher Forschung", in: Bausch 1982, S. 108-148.

Trudgill, Peter (1974): The social differentiation of English in Norwich. London 1974.

Werlen, Iwar (1986): Sprachbiographien von Ausländern der zweiten Generation. Arbeitsbericht zu einem soziolinguistischen Projekt. Universität Bonn, Institut für Sprachwissenschaft. Arbeitspapiere 20a und b, Bern 1986.

Wiesinger, Peter (1985): „Gesellschaftliche und sprachliche Probleme bei der Erforschung örtlicher Sprachgemeinschaften. Schwerpunkte der Forschungsgeschichte", in: Besch/Mattheier 1985, S. $24-48$.

Wildgen, Wolfgang (1986): „Synergetische Modelle in der Soziolinguistik. Zur Dynamik des 
Sprachwechsels Niederdeutsch-Hochdeutsch in Bremen um die Jahrhundertwende $(1880-1920)^{*}$, in: Zeitschrift für Sprachwissenschaft 5, 1986, S. 105-137.

Willis, Paul (1978): Profane culture. London 1978. Dt.: ,Profane Kultur. Rocker, Hippies: Subversive Stile der Jugendkultur. Frankfurt 1981.

Wirth, Louis (1928): The Ghetto. Chicago 1928.

Wirth, Louis (1938): „Urbanism as a way of life“, in: American journal of sociology 44, 1938 , S. 1-24. Dt.: „Die Stadt und die Zivilisation der Gegenwart“, in: Herlyn, U. (Hrsg.), Stadt- und Sozialstruktur. München 1974, S. 42-66.

Whyte, William F. (1955): Street corner society. Chicago 1955. 